\title{
Deformation and transfer doping of a single-walled carbon nanotube adsorbed on metallic substrates
}

\author{
Kazume Nishidate ${ }^{1, *}$ and Masayuki Hasegawa ${ }^{2}$ \\ ${ }^{1}$ Department of Electrical Engineering and Information Science, Iwate University, Morioka 020-8551, Japan \\ ${ }^{2}$ Faculty of Engineering, Iwate University, Morioka 020-8551, Japan
}

(Received 19 August 2009; revised manuscript received 14 January 2010; published 15 March 2010)

\begin{abstract}
We have examined the effects of radial deformation and transfer doping on the electronic properties of an armchair single-walled carbon nanotube (SWNT) adsorbed on the gold ( $\mathrm{Au})$ and silver $(\mathrm{Ag})$ surfaces. Using a semiempirical method developed on the basis of the continuum elastic shell model, it is found that the radial deformation of SWNTs with $D<21 \AA$, where $D$ is nanotube diameter, is reversible in the whole range of radial deformation. Whereas, large deformations of SWNTs with $D>21 \AA$ tend to be irreversible and a collapsed nanotube can be stabilized. We have chosen the metallic armchair $(21,21)$ SWNT with $D \approx 28.5 \AA$ and confirmed by $a b$ initio calculations that large deformation of this nanotube can actually be stabilized and the collapsed tube is a semiconductor with small band gap of $\sim 60 \mathrm{meV}$. The charge transfers of this nanotube, both circular and collapsed, adsorbed on the $\mathrm{Au}(100)$ and $\mathrm{Ag}(100)$ were investigated by large-scale $a b$ initio calculations and a phenomenological model, which was developed on the basis of the rigid-band model. The model yields the Fermi-level shift of the nanotube adsorbed on the Au(100) surface in good agreement with the experiments and provides a useful insight into the transfer doping. On the other hand, the transfer doping virtually does not occur for the nanotube adsorbed on the Ag surface, not in agreement with the experiments with large uncertainty. The phenomenological model is also applied to the graphene adsorption on the metallic surfaces and is found to yield reasonable results for the transfer doping and to be useful in understanding the previous results, both experimental and theoretical.
\end{abstract}

DOI: 10.1103/PhysRevB.81.125414

PACS number(s): 73.22.-f, 61.48.De, 61.46.Fg, 71.15.Mb

\section{INTRODUCTION}

Since the discovery ${ }^{1}$ of carbon nanotubes (CNTs), the electronic structures of individual single-walled CNTs (SWNTs) have been studied on the basis of how the band structure of the underlying graphitic sheet (graphene) is folded when the sheet is rolled up into a seamless cylinder. ${ }^{2-4}$ These results of the zone-folding model are modified by curvature effect ${ }^{5}$ and individual SWNTs can be classified according to their electronic band gap into semiconductors, small-gap semiconductors, and metals depending on size and chiraliry. In recent years, the structural, chemical, and environmental modifications of CNTs have attracted increasing attention with a motivation of finding possible technological applications. In particular, individual SWNTs are prone to a significant cross-sectional deformation under external stress as demonstrated by experiments ${ }^{6,7}$ and theoretical calculations. ${ }^{8-12}$ Of particular interest is that the band gaps of semiconductor SWNTs vary with increasing radial deformation and eventually close at a critical deformation, which depends on size and chirality. This electronic-structure modulation has been predicted theoretically ${ }^{13-21}$ and evidenced by experiments. ${ }^{22,23}$ On the other hand, the band-gap opening by deformation of metallic SWNTs, which is in contrast to the band-gap closing of semiconductor SWNTs, has been less investigated. ${ }^{13,24,25} \mathrm{Lu}$ et al. ${ }^{24}$ have argued that the combined effects of mirror-symmetry breaking and hybridization of $\pi$ states on the opposing flattened walls are responsible for the band-gap opening of armchair SWNTs. They demonstrated this electronic-structure transformation for the armchair $(8,8)$ SWNT using tight-binding (TB) molecular dynamics (MD) method. Mehrez et al. ${ }^{25}$ have also demonstrated this band-gap opening for a series of armchair $(n, n)$ SWNTs with $n=6-12$ using constraint-free densityfunctional TB-MD simulations. Their results show that armchair nonotubes with broken mirror symmetry can have zero band gap, implying that the mirror-symmetry breaking alone is insufficient for substantial band-gap opening of metallic SWNTs.

These electronic properties of free-standing SWNTs can be modified by the adsorption on the substrates, metal contact, adsorption of foreign chemical species, and other external disturbances. In particular, the use of a substrate as well as metal contact is unavoidable in most experiments and possible device applications. The substrates used in theoretical investigations and experimental scanning-tunneling spectroscopy (STS) studies include metals, ${ }^{26-35}$ semiconductors, ${ }^{36-38}$ and insulators. ${ }^{35,39}$ The nature of charge transfers and resulting electronic-structure modifications of the nanotubes adsorbed on these substrates have been discussed on the basis of the work-function (WF) difference between the nanotube and substrate. In the present work we are primarily concerned with the radial deformation and transfer doping of relatively large metallic SWNTs, specifically, armchair $(21,21)$ nanotube adsorbed on the metallic surfaces. We show that large radial deformation of this nanotube is irreversible and the collapsed nanotube can actually be stabilized. It is also confirmed that this collapsed nanotube is a semiconductor with small band gap of $\sim 60 \mathrm{meV}$, which are consistent with the results for much smaller armchair SWNTs. ${ }^{13,24,25}$ This stabilization without external stress could be useful in wider range of device applications making use of electronicstructure modulations by deformation. The choice of this large armchair nanotube was also motivated by the recent 
experiments by Giusca et al., ${ }^{34}$ who have made STS measurements on the flattened nanotube on the gold surface, identified as an armchair (21,21) SWNT with diameter $\approx 28 \AA$, and found that this nanotube is metallic with finite density of states (DOS) at the Fermi level. It is confirmed that the $p$-type doping is responsible for this metallic nature of the collapsed nanotube adsorbed on the gold substrate. We consider the (100) surfaces of gold $(\mathrm{Au})$ and silver $(\mathrm{Ag})$ solids, whose WFs are found to be 5.47 and $4.70 \mathrm{eV}$, respectively, and compared to that of SWNTs, $4.48 \mathrm{eV}$, implying different natures of the transfer doping for these metal substrates. However, the WF difference alone cannot explain nature of transfer doping. In the present work, large-scale $a b$ initio calculations and a phenomenological model, which is developed on the basis of the rigid-band model, are used to clarify the charge transfer of the nanotube adsorbed on the $\mathrm{Au}(100)$ and $\operatorname{Ag}(100)$ surfaces. A preliminary account of the present work is found elsewhere. ${ }^{40}$

This paper is organized as follows. In the next section we present the radial deformation of the armchair $(21,21)$ SWNT predicted by a semiempirical method and the results of $a b$ initio electronic-structure calculations for the deformed nanotube. In Sec. III we present a model for the geometrical structures of this nanotube adsorbed on the metallic surfaces and show the charge-transfer characteristic obtained by $a b$ initio calculations and a phenomenological model. The final section is devoted to the conclusions.

\section{RADIAL DEFORMATION AND ELECTRONIC STRUCTURES: FREE-STANDING NANOTUBES}

\section{A. Radial deformation}

We considered radial deformations uniformly extended along the nanotube axis and predicted plausible crosssectional shapes using a semiempirical method developed on the basis the continuum elastic shell model, ${ }^{12}$ which is briefly summarized in the following. This method is based on the observation that the strain energy, defined as the energy increase due to curvature effect, of SWNTs is well represented by that of the classical result for the continuum elastic shell. In fact, it has been found by the density-functional theory (DFT) and molecular mechanic calculations that the strain energy per atom of undeformed SWNTs is well represented as $E_{\text {strain }}=\gamma / R_{0}^{2}$ even for small SWNTs with $D=2 R_{0} \sim 10 \AA$, where $D\left(R_{0}\right)$ is the tube diameter (radius) and $\gamma$ is a constant $\left(\sim 1.94 \mathrm{eV} / \AA^{2}\right)$ insensitive to $R_{0}$ and chirality of nanotube. ${ }^{12,41}$ By extending this result to a deformed SWNT, the deformation energy, defined as the strain energy per atom with respect to the corresponding circular nanotube, is given by

$$
\Delta E_{0}=\frac{1}{2 \pi R_{0}} \oint \frac{\gamma}{R(s)^{2}} d s-\frac{\gamma}{R_{0}^{2}},
$$

where $R(s)$ is the local curvature radius along the circumference of the deformed cross section perpendicular to the nanotube axis and the integration is performed along the circumference. Also included in the continuum elastic shell model is the effect of the interactions between atoms on the
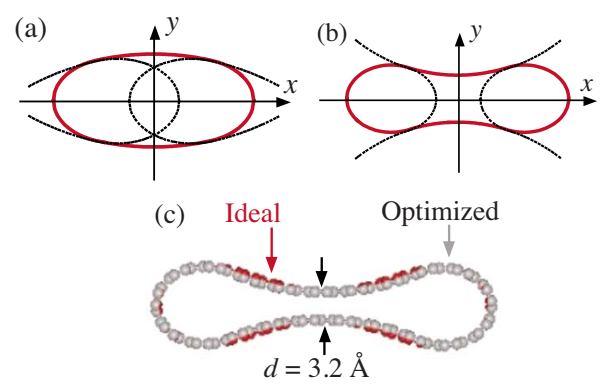

FIG. 1. (Color online) Model cross-sectional shapes of a radially deformed SWNT. The circumferences of (a) oval and (b) peanutlike shapes consist of two ellipsoids (on the edge parts) connected by two circles (on the top and bottom). Each shape transforms into another through a racetracklike shape, in which circular parts are parallel, straight lines. (c) Ideal (solid circles) and optimized (gray circles) cross-sectional structures of the collapsed armchair $(21,21)$ SWNT. The ideal one was obtained by conformal mapping of a graphene sheet on to the deformed cylindrical surface predicted by the continuum model.

opposing walls, which is not taken into account in Eq. (1). This effect is nominal for a small deformation but it is significant for a large enough deformation to make the minimum wall-wall distance comparable to the interlayer separation of graphite. This atomic interaction was obtained by a semiempirical analysis of the interlayer binding of graphite $^{42,43}$ and consists of the interaction reproducing the $a b$ initio DFT calculations in the local density approximation (LDA) and the van der Waals interaction not taken into account in the DFT calculations.

In the second step toward the prediction of radial deformation, we followed the previous work $^{12}$ and assumed a model cross-sectional shape whose circumference consists of two ellipsoids smoothly connected by two circles as illustrated in Fig. 1. This model shape contains four parameters and one of them can be eliminated by the assumption that the perimeter of a deformed cross section remains unchanged and is equal to $2 \pi R_{0}$. Then, for a given fractional change (decrease) in cross-sectional area $S$

$$
\eta=1-S / S_{0} \quad\left(S_{0}=\pi R_{0}^{2}\right)
$$

the shape parameters were determined so as to minimize the deformation energy, $\Delta E$, which consists of $\Delta E_{0}$ given by Eq. (1) and the contribution due to the wall-wall interaction. Since we assumed that no deformation occurs in the axial direction, $\eta$ also represents the fractional change in volume of a deformed nanotube and provides a useful parameter in treating radial deformation. In fact, if we assume that the model cross-sectional shape is consistent with an applied hydrostatic pressure, the pressure necessary to induce a deformation characterized by $\eta$ is proportional to $\partial \Delta E / \partial \eta$ and can easily be obtained. ${ }^{12}$

The results of calculations based on the above model indicate that the radial deformation of SWNTs with $D<21 \AA$ (Ref. 44) is reversible in the whole deformation regime unless the honeycomb structure of the nanotube is destroyed. Such a major atomic rearrangement has been predicted to occur for highly deformed nanotubes with 
$d<2.3 \AA,{ }^{25}$ where $d$ is the minimum wall-wall distance of a deformed nanotube. On the other hand, large deformations of SWNTs with $D>21 \AA$, typically thicker than the armchair $(15,15)$ and zigzag $(26,0)$ SWNTs, tend to be irreversible and a collapsed nanotube is stabilized under ambient pressure. This stabilization corresponds to the local minimum of $\Delta E$ as a function of $\eta$. Figure 1(c) shows a cross-sectional shape of the collapsed armchair $(21,21)$ SWNT with $D=28.5 \AA$ with which we are primarily concerned in the present work. The predicted cross-sectional shape of this collapsed nanotube is peanutlike with $d \approx 3.2 \AA$, slightly smaller than the graphitic interlayer separation $(3.4 \AA)$. Here, the atomic configuration was obtained by a conformal (ideal) mapping of a graphene sheet on to the deformed cylindrical surface predicted by the continuum model and, in that sense, no effect of atomic relaxation was taken into account. To check the validity and stability of the deformed cross-sectional shape obtained on the basis of the continuum model, we also performed structural optimization of the collapsed $(21,21)$ SWNT by the $a b$ initio DFT (LDA) method. All the DFT (LDA) calculations in the present work were performed using Vienna $a b$ initio simulation package (VASP) (Ref. 45) with the exchange-correlation energy functional of Ceperley and Alder $^{46}$ as parametrized by Perdew and Zunger. ${ }^{47}$ We used the projector-augmented wave $\operatorname{method}^{48}$ in modeling electron-ion interaction and the Monkhorst-Pack (MP) method $^{49}$ in the k-point sampling in the irreducible Brillouin zone. Varying approximations, depending on the type of calculations, were used for the k-point mesh in the MP method and the cut-off energy, $E_{\text {cut }}$, which limits the plane-wave expansion. In the structural optimization of the collapsed nanotube we used $E_{\text {cut }}=500 \mathrm{eV}$ and the $\mathbf{k}$ points generated from $1 \times 1 \times 9$ mesh. The atomic relaxation was made within the accuracy that the residual force acting on each atom decreases below $0.01 \mathrm{eV} / \AA$.

The optimized structure of the collapsed $(21,21)$ SWNT obtained by starting from the ideal atomic configuration is compared to the ideal one in Fig. 1(c). We find that both structures are quite similar to each other and the relaxation of each atom is also nominal, suggesting the validity and usefulness of the semiempirical method based the continuum model as well as the parameterized cross-sectional shape used in that method. The cross-sectional structure of a collapsed nanotube predicted by the present analyses [Fig. 1(c)] may be the most plausible among others proposed up to date. ${ }^{6-11} \mathrm{We}$ also note that geometrical optimization is generally irrelevant for large SWNTs, typically those with $D>15 \AA .{ }^{41,50}$ Our result indicates that this is also true for highly deformed and collapsed SWNTs.

\section{B. Electronic structure of armchair $(21,21)$ SWNT}

We considered armchair $(21,21)$ SWNT, which is the nanotube identified by Giusca et al. ${ }^{34}$ in their STS measurements on the collapsed nanotube. Following the prescription given in the previous section, the DFT (LDA) electronicstructure calculations were performed for this nanotube. Figure 2 shows the results for the undeformed (circular) and deformed nanotubes with atomic configurations obtained by
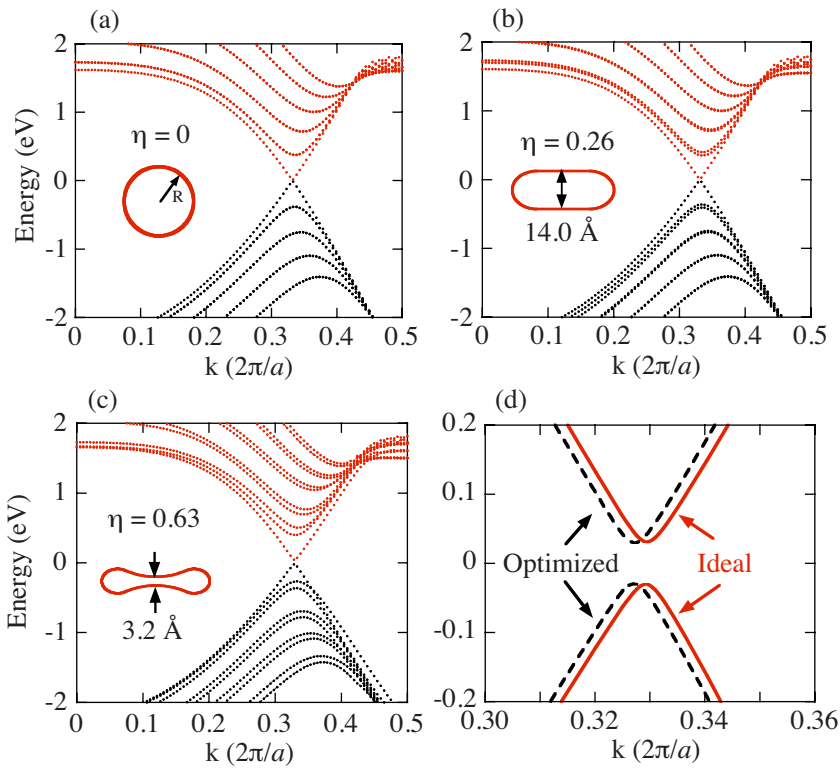

FIG. 2. (Color online) Electronic energy-band structures of the circular and deformed $(21,21)$ SWNTs with ideal atomic configurations obtained by rolling up a graphene sheet on to the cylindrical surface predicted by the continuum model. The Fermi-point energy is taken to be zero. (a) Circular nanotube (zero band gap), (b) highly deformed nanotube at $\eta=0.26$, the oval-to-peanut transition point (invisible band gap, if any), (c) collapsed nanotube (band gap of $\sim 60 \mathrm{meV}$ ), and (d) band structures near the Fermi point of the collapsed nanotube with ideal and optimized atomic structures illustrated in Fig. 1(c).

ideal mapping of carbon atoms on the deformed cylindrical surface. The armchair SWNTs with circular cross section are known to be real metals ${ }^{4}$ with $\pi-\pi^{*}$ band contact at the Fermi point, $k_{0} \approx(1 / 3)(2 \pi / a)$, where $a$ is the lattice constant of the honeycomb structure. This metallic nature remains unchanged and no tendency of band-gap opening is found even in the flattened nanotube with wall-wall distance of $d \approx 14 \AA$ [Fig. 2(b)]. The nanotube remains metallic up to a higher deformation at $\eta \sim 0.55(d \sim 5 \AA)$, beyond which this nanotube is collapsed spontaneously and stabilized at $\eta=0.63(d=3.2 \AA)$. The band gap of $E_{\mathrm{g}} \sim 60 \mathrm{meV}$ opens in the collapsed nanotube, indicative of semiconducting nature. These results demonstrate that appreciable band gap does not necessarily open in the armchair nanotubes with broken mirror symmetry, in accordance with the previous analyses for much smaller armchair SWNTs, ${ }^{25}$ and the $\pi-\pi$ interaction between the atoms on the opposing walls can be responsible for an appreciable band-gap opening. The effect of the structural optimization on the electronic structure was found to be nominal as demonstrated in Fig. 2(d) for the collapsed nanotube, which might have already been anticipated from the results in Fig. 1(c). In fact, the band gap virtually remains unchanged by the structural optimization, although the Fermi point slightly moves towards lower $k$, which is probably due to a small change in geometrical structure. Summarizing these results, we may conclude that deformed cross section alone, including its mirror-symmetry breaking, cannot induce an appreciable band gap of armchair SWNTs, and the minimum distance between flattened walls is responsible for the 
substantial band-gap opening through the $\pi$ - $\pi$ interaction.

The predicted band-gap opening of the collapsed $(21,21)$ SWNT is in contradiction with the STS measurements by Giusca et al., ${ }^{34}$ who showed that the collapsed $(21,21)$ nanotube adsorbed on the gold surface is metallic as a whole. In more detail, they observed that the flat region shows a metallic nature with finite local DOS at the Fermi level while the band gap opens in the edge region at either side of the flattened tube, indicative of a semiconducting nature. These observations for the armchair SWNT are in marked contrast to the implications found for the free-standing zigzag SWNTs, ${ }^{18,19,21}$ which show the reversed natures of the flat and edge regions. This difference between the armchair and zigzag SWNTs arises from the different natures of the bandedge state near the Fermi point. Here, we also note that the nanotube was collapsed by the tip of an atomic force microscope on the gold substrate in the experiments by Giusca et al. We show in the next section that the contradiction between the results for the free-standing nanotube and the experiments on the adsorbed nanotube can be explained by the effect of transfer doping.

\section{ADSORPTION OF AN ARMCHAIR NANOTUBE ON THE METALLIC SURFACES}

We considered the armchair $(21,21)$ SWNT, both undeformed (circular) and collapsed, adsorbed on the $\mathrm{Au}(100)$ and $\operatorname{Ag}(100)$ surfaces. These metal substrates have often been used in experiments and could be useful in some devise applications. The chemical properties and electronic structures of these metals are quite similar but the WF of $\mathrm{Au}$ is much larger than that of $\mathrm{Ag}$, implying different natures of transfer doping of the nanotube when adsorbed on these metal surfaces. We also note that the lattice constants of these metals are comparable, which enables us to treat these metals as substrates on the same footing. We first performed DFT (LDA) calculations for the fcc solids of these metals to predict their lattice constants, $a_{\mathrm{M}}$. In these calculations we used $20 \times 20 \times 20 \mathrm{MP}$ mesh in the k-point sampling for both metals, and $E_{\text {cut }}=287.3$ and $312 \mathrm{eV}$ for $\mathrm{Au}$ and $\mathrm{Ag}$, respectively. The predicted lattice constants were $a_{\mathrm{M}}=4.057$ and $4.013 \AA$ for $\mathrm{Au}$ and $\mathrm{Ag}$, and are about $0.6 \%$ and $1.9 \%$ smaller than the experimental values, 4.08 and $4.09 \AA,{ }^{51}$ respectively. These results together with the WFs are summarized in Table I. Underestimation of lattice constant and overestimation of binding are common features of DFT (LDA) calculations.

\section{A. Modeling geometrical structures}

The $\mathrm{Au}(100)$ and $\mathrm{Ag}(100)$ surfaces were modeled by fivelayer slabs and these structures were fully optimized with fixed in-plane lattice constant predicted by the DFT (LDA) calculations (Table I). By this structural optimization we have slightly reduced outermost interlayer distances compared to the bulk value, $a_{\mathrm{M}} / 2$, of the (100) surface. Next we put the nanotube, whose structure was fully optimized beforehand as described in the previous section, on the substrate in such a way that the nanotube axis is parallel to one
TABLE I. Lattice parameters, $a$ and $a_{\mathrm{M}}$, and the WF, $W_{\mathrm{NT}}$ and $W_{\mathrm{M}}$, of the armchair $(21,21) \mathrm{NT}$ and the M substrates. The values in the parentheses for the metal substrates are experimental values, which were not used in the present analyses.

\begin{tabular}{lcc}
\hline \hline System & $\begin{array}{c}a, a_{\mathrm{M}} \\
(\AA)\end{array}$ & $\begin{array}{c}\mathrm{WF} \\
(\mathrm{eV})\end{array}$ \\
\hline Circular NT & 2.46 & 4.48 \\
Collapsed NT & 2.46 & 4.55 \\
$\mathrm{Au}(100)$ & $4.057\left(4.08^{\mathrm{a}}\right)$ & $5.47\left(5.47^{\mathrm{b}}\right)$ \\
$\mathrm{Ag}(110)$ & $4.013\left(4.09^{\mathrm{a}}\right)$ & $4.70\left(4.64^{\mathrm{b}}\right)$ \\
\hline \hline
\end{tabular}

${ }^{\mathrm{a} A s h c r o f t}$ and Mermin (Ref. 51).

${ }^{b}$ Michaelson (Ref. 52).

of the translation vectors of the (100) square lattice, say $y$ direction. In the construction of a periodic structure required for the electronic-structure calculations, we chose a supercell containing five unit cells of armchair SWNT. With this choice the supercell size in the $y$ direction is $L_{\mathrm{y}}=5 a=12.30 \AA$, where $a$ is the lattice constant of armchair SWNT and taken to be $2.46 \AA$, corresponding to the experimental C-C bond length of $1.42 \AA$. This value of $L_{\mathrm{y}}$ is close to the three times of $a_{\mathrm{M}}$, i.e., $3 a_{\mathrm{M}}=12.17$ and $12.04 \AA$ for $\mathrm{Au}$ and Ag, respectively, using the DFT (LDA) values of $a_{\mathrm{M}}$. This supercell construction provides the smallest possible $L_{\mathrm{y}}$ with tolerable lattice mismatch for armchair tubes adsorbed on the $\mathrm{Au}(100)$ and $\mathrm{Ag}(100)$ surfaces. In order to eliminate lattice mismatch and to achieve a complete periodicity, we stretched the $\mathrm{Au}$ and $\mathrm{Ag}$ slabs in the $y$ direction by $\sim 1 \%$ and $2 \%$, respectively, while the lattice constants in the lateral $x$ direction are fixed at the values given above. The supercell size in the lateral $x$ direction was chosen to accommodate thirteen surface unit cells, i.e., $L_{x}=13 a_{\mathrm{M}}=52.75$ and $52.17 \AA$ for the Au and Ag substrates, respectively. The supercell size in the surface-normal direction, $L_{z}$, was taken to be the same as $L_{x}$. With this supercell construction, the closest distance between the nanotubes in the neighboring cells is $\sim 12 \AA$ even for the collapsed tube and the vacuum gap over the nanotube is $\sim 10 \AA$ even for the circular nanotube, both being large enough to simulate an individual nanotube on the substrate. The supercell constructed in this way is virtually the smallest possible one for simulating armchair $(21,21)$ SWNT adsorbed on the $\mathrm{Au}(100)$ and $\mathrm{Ag}(100)$ surfaces. This supercell consists of 420 carbon atoms, 390 metal atoms, and so 810 atoms in total, implying that computations are inevitably quite demanding.

The final step in modeling a geometrical structure is to make structural optimization of the combined system consisting of the metallic slab and adsorbed nanotube. In this optimization, only the topmost three layers of the substrate were subject to relaxation while lower bottom layers were fixed. We found no appreciable structural changes in both the nanotube and substrate by this optimization, implying a weak coupling between them. In fact, the closest contact distances between the nanotube and substrate were, in all cases under consideration, $\sim 3.4 \AA$, which is very close to the graphitic interlayer separation and typical of physisorption involving carbon atoms. As we see below, electronic-structure 
calculations also show no indication of bond-forming tendency.

\section{B. Charge transfer: DFT calculations}

Charge transfers of the nanotubes adsorbed on the substrates have often been discussed in terms of the WF difference. $29,33,35,38,39$ The DFT results for the WFs of SWNTs with $D>10 \AA$ have been $4.5-4.7 \mathrm{eV}$, depending on the type of calculations and insensitive to size and chirality of nanotubes. ${ }^{53-56}$ The nanotube WFs have also been studied by photoemission electron spectroscopy ${ }^{57-59}$ but these experimental results are quantitatively somewhat uncertain. We also calculated WF of the armchair $(21,21)$ nanotube, both circular and collapsed, using $1 \times 1 \times 30 \mathrm{MP}$ k-point mesh and $E_{\text {cut }}=400 \mathrm{eV}$. Our result for the circular nanotube, $W_{\mathrm{NT}}=4.48 \mathrm{eV}$, is in good agreement with the previous calculations ${ }^{55,56}$ and is close to the graphene limit. We found a slightly larger WF for the collapsed nanotube, $W_{\mathrm{NT}}=4.55 \mathrm{eV}$. These results are summarized in Table I. The WFs of the $\mathrm{Au}(100)$ and $\mathrm{Ag}(100)$ surfaces, modeled by slabs, were also calculated using $9 \times 9 \times 1 \mathrm{MP}$ k-point mesh with $E_{\text {cut }}=300 \mathrm{eV}$ and were found to be 5.47 and $4.70 \mathrm{eV}$, respectively, in good agreement with experiments (Table I). ${ }^{52}$ These values are by $\sim 1.0$ and $0.2 \mathrm{eV}$ larger than that of the nanotube, respectively. We also calculated the WFs of these metal substrates stretched by $3 \%$ to see the effect of artificial strain on the charge transfer and found $\sim 0.1 \mathrm{eV}$ increase for $\mathrm{Au}$ and negligible change for Ag. These degrees of change may be ignored in the following analyses of the charge transfer. We also expect as usually that other electronic properties, which might have an influence on the charge transfer, may not be affected by the stretch or compression of a few percent.

The charge densities were calculated for the systems of the circular and collapsed $(21,21)$ nanotubes adsorbed on the $\mathrm{Au}(100)$ and $\operatorname{Ag}(100)$ substrates with fully optimized structures. In these DFT (LDA) calculations with $E_{\text {cut }}=300 \mathrm{eV}$, we used only the $\Gamma$ point in the k-point sampling to avoid computational difficulty in dealing with a huge number of wave functions in the large supercell calculations. ${ }^{60}$ Following the common practice, ${ }^{33}$ we can visualize charge redistribution using the difference density defined by

$$
\Delta n(\mathbf{r})=n(\mathbf{r})-n_{\mathrm{NT}}(\mathbf{r})-n_{\mathrm{M}}(\mathbf{r}),
$$

where $n(\mathbf{r})$ is the electron number density of the combined system consisting of the nanotube (NT) and metallic (M) substrate, and $n_{\mathrm{NT}}(\mathbf{r})\left[n_{\mathrm{M}}(\mathbf{r})\right]$ is that of the nanotube (substrate) obtained by simply removing the substrate (nonotube) from the atomic configuration of the combined system. Figure 3 illustrates the prominent regions of $\Delta n(\mathbf{r})<0$ and $\Delta n(\mathbf{r})>0$ for the systems of NT/Au(100) and NT/Ag(100). Illustrated in this figure are the isosurfaces with $|\Delta n(\mathbf{r})| /|\Delta n(\mathbf{r})|_{\max }=0.05$, where $|\Delta n(\mathbf{r})|_{\max }$ is the maximum value in each region, so electron deficiency $[\Delta n(\mathbf{r})<0]$ and excess $[\Delta n(\mathbf{r})>0]$ occur mostly in the regions confined to those isosurfaces. The results in Fig. 3 indicate that electrons are mostly removed from the nanotube and accumulate in the interfacial region between the nanotube and substrate while

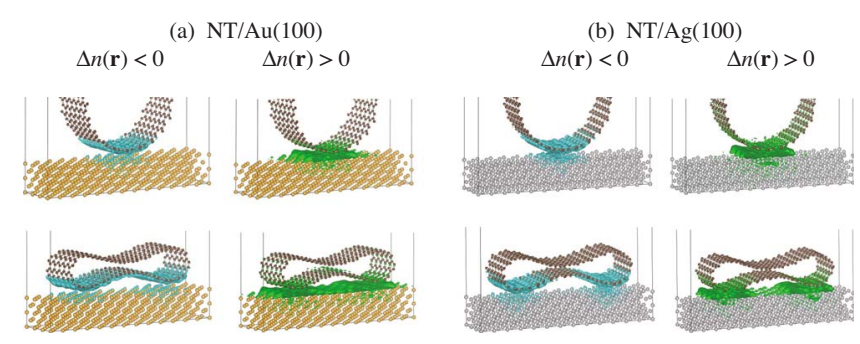

FIG. 3. (Color online) Charge redistributions in the systems of circular and collapsed $(21,21)$ SWNTs adsorbed on the $\mathrm{Au}(100)$ and $\operatorname{Ag}(100)$ surfaces. The shaded areas show the isosurfaces with $|\Delta n(\mathbf{r})| /|\Delta n(\mathbf{r})|_{\max }=0.05$ in the regions of $\Delta n(\mathbf{r})<0$ (left panel) and $\Delta n(\mathbf{r})>0$ (right panel), where $\Delta n(\mathbf{r})$ is defined by Eq. (5) and $|\Delta n(\mathbf{r})|_{\max }$ is the maximum value in each region. The electron deficiency $(\Delta n(\mathbf{r})<0)$ and excess $(\Delta n(\mathbf{r})>0)$ occur mostly in the regions confined to these isosurfaces.

electrons are also removed, to lesser extent, from the metallic substrate. These charge redistributions can be seen more clearly using the plane-averaged difference density, which is obtained by integrating $\Delta n(\mathbf{r})$ over the lateral plane

$$
\Delta N(z)=\iint \Delta n(\mathbf{r}) d x d y .
$$

The results of $\Delta N(z)$ for the circular and collapsed nanotubes on the metallic surfaces are illustrated in Fig. 4. The atomic positions with the closest contact are also shown in this figure and, as we have pointed out earlier, the distances between them are $\sim 3.4 \AA$ in all cases under consideration. We find only small difference between the results for NT/ $\mathrm{Au}(100)$ and NT/Ag(100), indicating that, contrary to a general perception, the WF difference, $\Delta W=W_{\mathrm{M}}-W_{\mathrm{NT}}$, does not necessarily play a role of motive force for this charge redistribution. Here, $\Delta W \sim 1.0$ and $0.2 \mathrm{eV}$ for NT/Au(100) and NT/Ag(100), respectively (Table I). These results for the nanotube are similar to those of the graphene absorbed on the $\mathrm{Au}$ and Ag substrates. ${ }^{62}$ As we discuss later, this "charge redistribution" is different from the "charge transfer," which is defined in terms of the Fermi-level shift within the rigidband model. We find that the charge redistributions are more
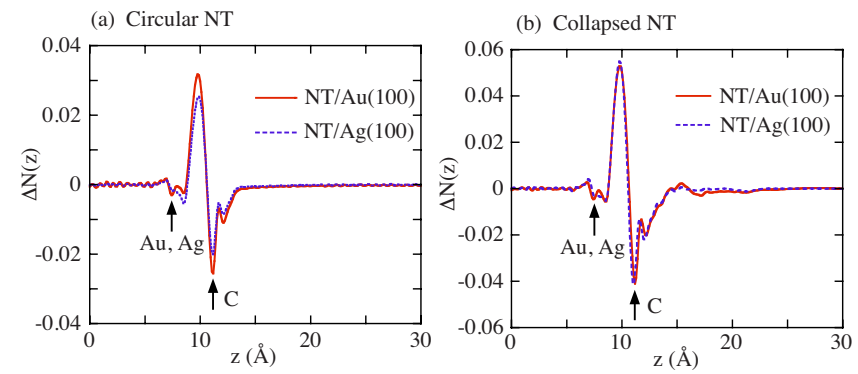

FIG. 4. (Color online) Plane-averaged difference number densities obtained by integrating $\Delta n(\mathbf{r})$ over the lateral plane [Eq. (6)]. The unit of $\Delta N(z)$ is number $/ \Delta z$ with $\Delta z=L_{z} / 448, L_{z}$ being the supercell size in the $z$ direction (perpendicular to the substrate surface). Vertical arrows show the atomic positions of the closest contact between the substrate and nanotube, and the distance between them is $\sim 3.4 \AA$ in all the cases under consideration. 
TABLE II. Summary of the parameters for the armchair $(21,21)$ NT adsorbed on the $\mathrm{Au}(100)$ and $\mathrm{Ag}(100)$ surfaces. $N_{\text {re }}$ is defined by Eq. (5) and represents the number of electrons (per supercell containing 420 carbon atoms) removed from the nanotube by the charge redistribution. $\Delta W$ is the work-function difference used to calculate the Fermi-level shift, $\Delta E_{\mathrm{F}}$, and the number of transferred electrons (per supercell), $N_{\mathrm{tr}} . \Delta V_{\mathrm{c}}$ and $\Delta V_{\mathrm{NT}}$ are the potential steps defined in Eqs. (13) and (14). The values in the parentheses represent the results from the DFT (LDA) calculations (Fig. 5).

\begin{tabular}{lcccccc}
\hline \hline System & $N_{\mathrm{re}}$ & $\begin{array}{l}\Delta W \\
(\mathrm{eV})\end{array}$ & $\begin{array}{l}\Delta E_{\mathrm{F}} \\
(\mathrm{eV})\end{array}$ & $N_{\mathrm{tr}}$ & $\begin{array}{c}\Delta V_{\mathrm{c}} \\
(\mathrm{eV})\end{array}$ & $\begin{array}{c}\Delta V_{\mathrm{NT}} \\
(\mathrm{eV})\end{array}$ \\
\hline Circular-NT/Au & 0.56 & 0.99 & $0.29(0.75)$ & $0.84(0.25)$ & 0.13 & 0.80 \\
Collapsed-NT/Au & 0.87 & 0.92 & $0.26(0.64)$ & $0.83(0.20)$ & 0.15 & 0.75 \\
Circular-NT/Ag & 0.41 & 0.22 & $0.03(0.04)$ & $0.09(\sim 0)$ & 0.14 & 0.07 \\
Collapsed-NT/Ag & 0.87 & 0.15 & $-0.03(-0.02)$ & $\sim 0(\sim 0)$ & 0.18 & -0.09 \\
\hline \hline
\end{tabular}

extensive for the collapsed nanotube, which might be explained by larger contact area in that case.

The total number of electrons removed from the nanotube, $N_{\text {re }}$, was estimated by integrating $\Delta N(z)$ over the region of the nanotube $\left(z>z_{0}\right)$ (Ref. 62)

$$
N_{\text {re }}=-\int_{z_{0}}^{\infty} \Delta N(z) d z .
$$

Here, $z_{0}$ was taken to be the nodal point of $\Delta N(z)$ just outside the nanotube although this choice is somewhat arbitrary (see Fig. 4). With this choice of $z_{0}$ we find that $N_{\text {re }}=0.56$ and 0.41 for the circular nanotube on the $\mathrm{Au}(100)$ and $\mathrm{Ag}(100)$ surfaces, respectively, and $N_{\text {re }}=0.87$ for the collapsed nanotube on both surfaces. These results of $N_{\text {re }}$, summarized in Table II, represent the numbers per unit supercell with carbon atoms of $N_{\mathrm{C}}=420$ and so are effectively quite small. In fact, we find $N_{\text {re }} / N_{\mathrm{C}}=0.0021$ per carbon atom for the collapsed nanotube, which is compared to the corresponding values of $\sim 0.008$ for the graphene adsorbed on the $\mathrm{Au}$ and $\mathrm{Ag}$ surfaces. ${ }^{62}$ Both results for the nanotube and graphene are effectively comparable because, in the case of the nanotube adsorption, only the limited number of carbon atoms, one forth or less, are in contact with the substrate and so are involved in the charge redistribution. The positive values of $N_{\text {re }}$ for both surfaces might be consistent with the $p$-type doping of the nanotube with $\Delta W>0$, which indicates that the absolute Fermi energy of the substrate is lower than that of the nanotube. However, we note again that the definition of $N_{\text {re }}$ is somewhat arbitrary, and, more importantly, the type of doping and its heaviness are almost independent of $N_{\text {re }}$, as we discuss later.

As we have noted earlier, geometrical structure of the nanotube shows no appreciable change by the adsorption on the substrate with the closest contact distance typical of physisorption. The charge redistributions also imply that the coupling between the nanotube and substrates is weak with no bond-forming tendency. In such a situation, the electronic structure of the adsorbed nanotube may be more or less preserved as actually suggested by STS measurements on a similar system ${ }^{32}$ and such a rigid-band nature may be tested in terms of the DOSs. Figure 5 shows the DOSs of the circular nanotube, metallic substrate, and the combined system of the nanotube and substrate, which we denote $\rho_{\mathrm{NT}}(E)$,
$\rho_{\mathrm{M}}(E)$, and $\rho(E)$, respectively. These DOSs correspond to the charge densities defined in Eq. (3) and were obtained by the same calculations, in which only the $\Gamma$ point was used in the $k$-point sampling for avoiding computational difficulty. The Fermi energy of each system is taken to be zero in Figs. 5(a) and 5(b). No singular behavior is found in these DOSs because the Gaussian smearing of $0.2 \mathrm{eV}$ width was used in the electronic-structure calculations. Highly enhanced $\rho(E)$ and $\rho_{\mathrm{M}}(E)$ below the Fermi level show the contributions due to the valence $d$ states of the metallic substrates. The rigid-band nature can clearly be seen using a superimposed DOS defined by
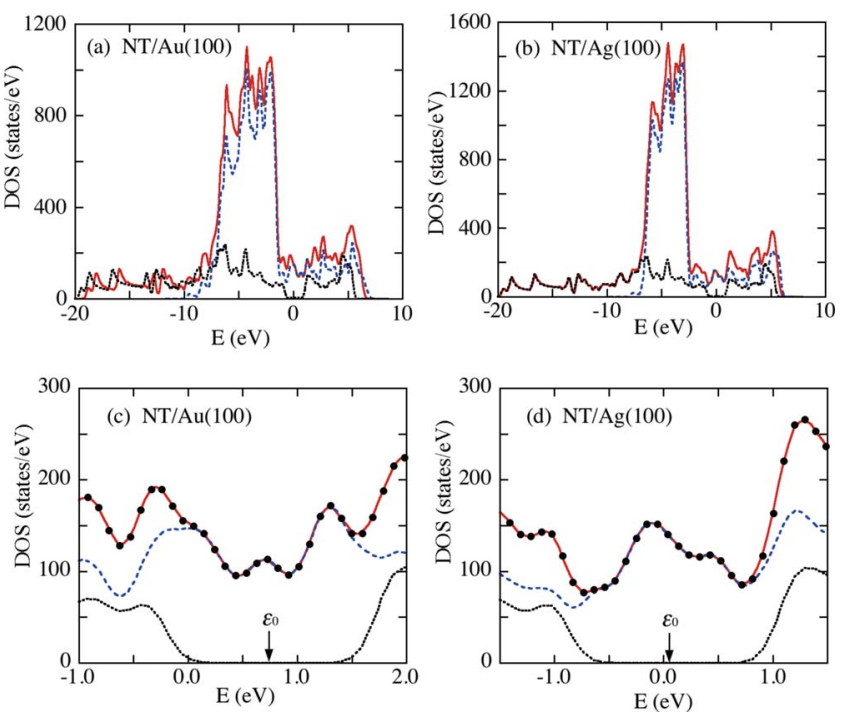

FIG. 5. (Color online) DOSs of the systems of circular NT adsorbed on the $\mathrm{Au}(100)$ and $\mathrm{Ag}(100)$ surfaces. Solid, dashed, and dotted lines show the DOSs of the combined system $[\rho(E)]$, metallic substrate $\left[\rho_{\mathrm{M}}(E)\right]$, and adsorbed nanotube $\left[\rho_{\mathrm{NT}}(E)\right]$, respectively. In (a) and (b), the Fermi energy of each system is taken to be zero. In (c) and (d), the aligned Fermi energy of the combined system is taken to be zero and the solid circles show the superimposed DOS, $\rho_{\text {rigid }}(E)$, obtained by superimposing appropriately shifted $\rho_{\mathrm{NT}}(E)$ and $\rho_{\mathrm{M}}(E)$ [Eq. (6)]. Vertical arrows show the position of the Fermi point of the nanotube. 


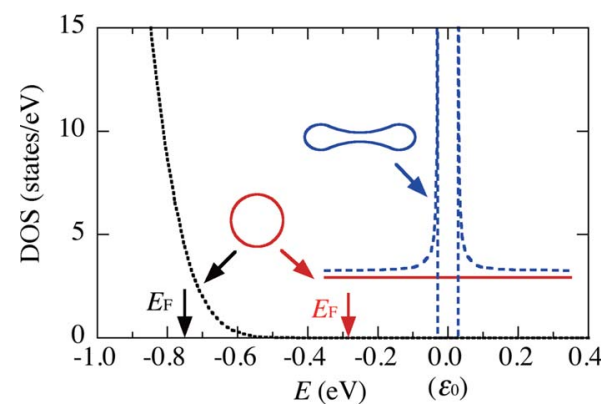

FIG. 6. (Color online) DOSs per supercell of the circular and collapsed $(21,21)$ SWNTs adsorbed on the $\mathrm{Au}(100)$ surface. The dotted line shows the DOS obtained by the DFT (LDA) calculations [Fig. 5(c)] and solid and dashed lines show the DOSs obtained by the accurate band structure calculations [Figs. 2(a) and 2(c)]. The Fermi-point energy $\left(\varepsilon_{0}\right)$ of the nanotube is taken to be zero and the vertical arrows show the positions of the Fermi energy, $E_{\mathrm{F}}$, corresponding to the DOSs (solid and dotted lines) of the circular nanotube on the $\mathrm{Au}(100)$. The Fermi-level shift defined by $\Delta E_{\mathrm{F}}=\varepsilon_{0}-E_{\mathrm{F}}$ and the number of transferred electrons, $\Delta N_{\mathrm{tr}}$, are summarized in Table II.

$$
\rho_{\text {rigid }}(E)=\rho_{\mathrm{NT}}\left(E-\Delta E_{\mathrm{NT}}\right)+\rho_{\mathrm{M}}\left(E-\Delta E_{\mathrm{M}}\right) .
$$

Here, $\rho_{\mathrm{NT}}(E)$ and $\rho_{\mathrm{M}}(E)$ are appropriately shifted by $\Delta E_{\mathrm{NT}}$ and $\Delta E_{\mathrm{M}}$, respectively, toward higher energies and are superimposed to define $\rho_{\text {rigid }}(E)$. The energy shifts, $\Delta E_{\mathrm{NT}}$ and $\Delta E_{\mathrm{M}}$, can be interpreted as the Fermi-level shifts of the nanotube and substrate relative to that of the combined system. As illustrated in Figs. 5(c) and 5(d), $\rho_{\text {rigid }}(E)$ can almost completely be fit to $\rho(E)$ by appropriately choosing $\Delta E_{\mathrm{NT}}$ and $\Delta E_{\mathrm{M}}$, indicating that the rigid-band picture is valid. These fits can be achieved using very small values of $\Delta E_{\mathrm{M}}$, typically $\sim 0.01 \mathrm{eV}$, which may be taken to be zero within the numerical accuracy. This result can be explained by that the DOS near the Fermi level of a nanotube is quite small compared to that of the metallic substrates and so the doping effect on the Fermi-level shift is effective only for the nanotube. We found that $\Delta E_{\mathrm{NT}}=0.75$ and $0.04 \mathrm{eV}$ for the circular nanotube adsorbed on the $\mathrm{Au}(100)$ and $\operatorname{Ag}(100)$ surfaces, respectively. The superimposed DOS, $\rho_{\text {rigid }}(E)$, obtained using these parameters are actually well compared to $\rho(E)$ [Figs. 5(c) and 5(d)]. Similar results were obtained for the collapsed nanotube and the results of the Fermi-level shift, $\Delta E_{\mathrm{NT}}$, which we denote $\Delta E_{\mathrm{F}}$ hereafter, are summarized in Table II.

The above DFT (LDA) result, $\Delta E_{\mathrm{F}}=0.75 \mathrm{eV}$, for the circular- $\mathrm{NT} / \mathrm{Au}(100)$ is a few times as large as the experiments ${ }^{26,30}$ and the previous theoretical predictions. ${ }^{29}$ This discrepancy is the consequence of the unrealistic DOS obtained by the simplified DFT calculations, which were inevitable for a practical reason. In fact, the nanotube DOS near the Fermi point virtually vanishes in those calculations (Fig. 5) and the electrons to be transferred from the nanotube to the substrate are not available in that region. Such electrons are available in the region of much lower energy, where the DOS due to the tail of the Gaussian-smeared second subband comes into play as illustrated in Fig. 6. The numbers of

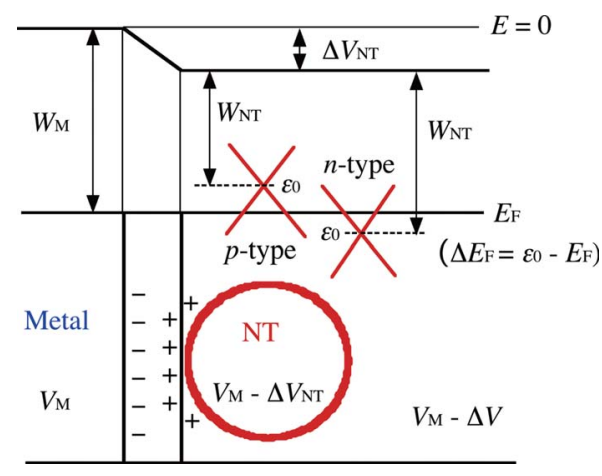

FIG. 7. (Color online) Schematic representation of the Fermienergy alignment [Eq. (13)]. The $p$-type doping of the NT is assumed for the distribution of the transferred charges.

electrons, $N_{\text {tr }}$, transferred from the nanotube to the substrate is given by

$$
N_{\mathrm{tr}}=-\int_{\varepsilon_{0}}^{E_{\mathrm{F}}} \rho_{\mathrm{NT}}(E) d E
$$

within the rigid-band model. Using the DOSs and the Fermilevel shifts obtained by the DFT (LDA) calculations in Eq. (7), the values of $N_{\text {tr }}$ were estimated to be 0.247 and 0.202 (states/eV)/supercell for the circular-NT/Au(100) and collapsed-NT/Au(100), respectively. These values are much smaller than those of $N_{\text {re }}$ obtained from the charge redistributions. Charge transfer virtually does not occur and we have very small Fermi-level shift for the NT/Ag(100), which also differ from finite, relatively large $N_{\text {re }}$. For the NT/Ag(100) we expect a small amount of charge transfer because of the small WF difference $(\Delta W \sim 0.2 \mathrm{eV})$ but the DOS in the vicinity of the Fermi point virtually vanishes, implying $N_{\text {tr }} \sim 0$. In this way, these results of $\Delta E_{\mathrm{F}}$ and $N_{\text {tr }}$ obtained by the simplified DFT calculations may be unrealistic. However, such DFT calculations provide a model system with particular DOS, which is useful and can actually be utilized in the analyses of the charge transfer. We actually show the limitation and usefulness of such DFT calculations in the analyses based on the phenomenological model (in Sec. III D).

\section{Rigid-band model}

Charge transfer of the nanotube adsorbed on the substrate is defined, within the rigid-band model, by Eq. (7) and the Fermi-level shift, $\Delta E_{\mathrm{F}}=\varepsilon_{0}-E_{\mathrm{F}}$, where $\varepsilon_{0}$ and $E_{\mathrm{F}}$ are, as before, the Fermi-point energy of the free-standing nanotube and the Fermi energy of the combined system, respectively. Figure 7 shows a schematic representation of the Fermienergy alignment, which is discussed in more detail in the next section. With these definitions, $\Delta E_{\mathrm{F}}$ and $N_{\mathrm{tr}}$ are positive (negative) for the $p$-type ( $n$-type) doping of the nanotube and these quantities can explicitly be related to each other using a realistic $\rho_{\mathrm{NT}}(E)$ as we show in the following.

The energy band near the Fermi point $\left(k_{0}, \varepsilon_{0}\right)$ of an armchair circular nanotube is given by $E(k)=\varepsilon_{0} \pm \beta_{0}\left|k-k_{0}\right|$, as actually obtained by the DFT calculations for the $(21,21)$ SWNT [Fig. 2(a)]. Then, the DOS per atom of an armchair $(n, n)$ nanotube is given by ${ }^{61}$ 


$$
\rho_{\mathrm{NT}}(E)=(a / n \pi)|d E / d k|^{-1}=(2 / n)\left(2 \pi \beta_{0} / a\right)^{-1} \equiv \rho_{0} .
$$

The effect of charge transfer is, depending on the sign of $N_{\text {tr }}$, either to make the bottom part of the $\pi^{*}$-band occupied (n-type doping) or to make the top part of the $\pi$-band unoccupied ( $p$-type doping). If only the linear bands are involved $\left(\left|\Delta E_{\mathrm{F}}\right|<0.35 \mathrm{eV}\right.$ for the $(21,21)$ nanotube), we have $N_{\text {tr }}=\rho_{0} \Delta E_{\mathrm{F}}$, where $N_{\text {tr }}$ is now defined as the number per atom. We find $2 \pi \beta_{0} / a=13.7 \mathrm{eV}$ from the result in Fig. 2(a) and hence $\rho_{0}=6.95 \times 10^{-3} / \mathrm{eV}$. On the other hand, for the collapsed $(n, n)$ nanotube with small band gap [Fig. 2(c)], the band dispersion near the Fermi point is to a good approximation given by

$$
E(k)=\varepsilon_{0} \pm \sqrt{\beta^{2}\left(k-k_{0}\right)^{2}+\left(E_{\mathrm{g}} / 2\right)^{2}}
$$

and the DOS by

$$
\begin{aligned}
\rho_{\mathrm{NT}}(E) & =\frac{2}{n} \frac{a}{2 \pi \beta} \frac{\left|E-\varepsilon_{0}\right|}{\sqrt{\left(E-\varepsilon_{0}\right)^{2}-\left(E_{\mathrm{g}} / 2\right)^{2}}}\left(\left|E-\varepsilon_{0}\right| \geq E_{\mathrm{g}} / 2\right) \\
& =0\left(\left|E-\varepsilon_{0}\right|<E_{\mathrm{g}} / 2\right) .
\end{aligned}
$$

Then, assuming that only the energy band given by Eq. (9) is involved, we have

$$
N_{\mathrm{tr}}= \pm \frac{2}{n} \frac{a}{2 \pi \beta} \sqrt{\left(\Delta E_{\mathrm{F}}\right)^{2}-\left(E_{\mathrm{g}} / 2\right)^{2}} \quad\left(\left|\Delta E_{\mathrm{F}}\right|>E_{\mathrm{g}} / 2\right),
$$

where the sign $( \pm)$ corresponds to that of $\Delta E_{\mathrm{F}}$ (i.e., the signs of $N_{\text {tr }}$ and $\Delta E_{\mathrm{F}}$ are the same). Equation (11) can be rewritten as

$$
\Delta E_{\mathrm{F}}= \pm \sqrt{\left(E_{\mathrm{g}} / 2\right)^{2}+(n / 2)^{2}(2 \pi \beta / a)^{2} N_{\mathrm{tr}}^{2}} .
$$

For the collapsed $(21,21)$ nanotube, we find $2 \pi \beta / a=12.3 \mathrm{eV}$ from the results in Fig. 1(c), slightly smaller than the corresponding one of the circular nanotube. For the $(21,21)$ nanotube, the DOS due to other bands (subbands) must be taken into account in Eqs. (10) and (11) if $\left|\Delta E_{\mathrm{F}}\right|>0.35 \mathrm{eV}$ (see Fig. 2). These expressions for $N_{\text {tr }}$ and $\Delta E_{\mathrm{F}}$ for the realistic DOS may be conveniently used in the following analyses of the Fermi-level shift.

\section{Charge transfer: Phenomenological model}

The Fermi-energy alignment, which is schematically represented in Fig. 7, was already implicit in Figs. 5(c) and 5(d). In Fig. 7, $p$-type doping of the nanotube is assumed for the charge distribution. Following Khomyakov et al., ${ }^{62}$ who have studied graphene adsorption on the metallic surfaces, we define $\Delta V_{\mathrm{NT}}$ and $\Delta V$ as the down-ward potential steps, with respect to the substrate, in the nanotube and in the vacuum region far from the nanotube, respectively. Both $\Delta V_{\mathrm{NT}}$ and $\Delta V$ are the potential steps due to the interaction between the nanotube and substrate although they are not so well defined as in the case of the graphene adsorption on the metallic surfaces. The reason for this is that the interaction between the nanotube and substrate is not uniform over the interface, and both $\Delta V_{\mathrm{NT}}$ and $\Delta V$ must be interpreted as averaged quantities. Then, the condition of the Fermi-energy alignment can be written as ${ }^{63}$

$$
W_{\mathrm{M}}=W_{\mathrm{NT}}+\Delta E_{\mathrm{F}}+\Delta V_{\mathrm{NT}}=W+\Delta V .
$$

Here, $\Delta E_{\mathrm{F}}$ is the Fermi-level shift as before and $W$ is the WF of the substrate with nanotube on the surface. As we have pointed out earlier, we assumed in Eq. (13) that the Fermilevel shift of the substrate is negligible $\left(\Delta E_{\mathrm{M}} \approx 0\right)$. If the contact area is small compared to the substrate surface area, the potential step, $\Delta V$, is expected to be small and even negligible. In other words, $W$ is close to $W_{\mathrm{M}}$. We actually confirmed that $W$ is slightly, by $\sim 0.01 \mathrm{eV}$ at most, smaller than $W_{\mathrm{M}}$, implying that $\Delta V<0.01 \mathrm{eV}$. Hence, as we have already assumed in the analyses of the DOSs (Figs. 5 and 6), $\Delta V$ is set equal to zero in the following analyses. The finite value of $\Delta V$ may be interpreted as an effect of finiteness of the supercell size in the lateral direction.

Following Khomyakov et al., ${ }^{62}$ we also consider a phenomenological model, which is similar to that proposed by them for the graphene adsorption on the metallic surfaces. The model starts with writing the interface potential step as

$$
\Delta V_{\mathrm{NT}}=\Delta V_{\mathrm{c}}+\Delta V_{\mathrm{tr}} .
$$

The first term, $\Delta V_{c}$, describes the short-range repulsion resulting from the overlap of the wave functions and depends primarily on the distance between the atoms of the nanotube and metallic substrate. The second term, $\Delta V_{\text {tr }}$, represents the potential step directly induced by the charge transfer and may be proportional to the number of transferred electrons, $\Delta V_{\mathrm{tr}}=\alpha N_{\mathrm{tr}}$, where $\alpha$ is a constant depending on the spatial distribution of the transferred electrons and $N_{\mathrm{tr}}$ can be written as in Eq. (7). This form of $\Delta V_{\text {tr }}$ is crucial in the present model. Both $\Delta V_{\mathrm{c}}$ and $\Delta V_{\text {tr }}$ must be interpreted as the averaged quantities as mentioned before. With the above form of $\Delta V_{\text {tr }}$, the energy increase, $d U_{\text {tr }}$, due to the increase by $d N_{\text {tr }}$ of $N_{\text {tr }}$, is the sum of the energy cost for the transferred $d N_{\text {tr }}$ electrons to overcome the barrier height, $\Delta V_{\mathrm{tr}}=\alpha N_{\mathrm{tr}}$, and their potential-energy loss of $-\Delta V_{\mathrm{tr}}=-\alpha N_{\mathrm{tr}}$

$$
d U_{\text {tr }}=d N_{\text {tr }} \alpha N_{\text {tr }}-d N_{\text {tr }}\left(-\alpha N_{\text {tr }}\right)=2 \alpha N_{\text {tr }} d N_{\text {tr }} .
$$

Then, the energy cost to transfer $N_{\text {tr }}$ electrons to the substrate is given by

$$
U_{\mathrm{tr}}=2 \alpha \int_{0}^{N_{\mathrm{tr}}} N_{\mathrm{tr}}^{\prime} d N_{\mathrm{tr}}^{\prime}=N_{\mathrm{tr}} \Delta V_{\mathrm{tr}} .
$$

The total energy increase by the charge transfer is given by the sum of $U_{\text {tr }}$ and the energy gain due to the doping, $U_{\text {doping }}$, in which transferred electrons are on the aligned Fermi level, $E_{\mathrm{F}}$

$$
U=\int_{\varepsilon_{0}}^{E_{\mathrm{F}}}\left(E-E_{\mathrm{F}}\right) \rho_{\mathrm{NT}}(E) d E+N_{\mathrm{tr}} \Delta V_{\mathrm{tr}} .
$$

The Fermi-level shift, $\Delta E_{\mathrm{F}}=\varepsilon_{0}-E_{\mathrm{F}}$, or $N_{\text {tr }}$ given by Eq. (7) may be determined by the requirement that $U$ is minimum with respect to $\Delta E_{\mathrm{F}}$ under the constraint of the Fermi-energy alignment as given by Eq. (13). We solved this optimization problem using the Lagrange multiplier method and found that $\Delta E_{\mathrm{F}}$ is determined by the equation (the derivation is given in Appendix) 


$$
\Delta E_{\mathrm{F}}+2 \frac{N_{\mathrm{tr}}\left(\Delta E_{\mathrm{F}}\right)}{\rho_{\mathrm{NT}}\left(E_{\mathrm{F}}\right)}=\Delta W-\Delta V_{\mathrm{c}} .
$$

If the Fermi energy $E_{\mathrm{F}}$ is in the range of the linear bands of a circular armchair nanotube, we have $N_{\mathrm{tr}}\left(\Delta E_{\mathrm{F}}\right) \approx \rho_{\mathrm{NT}}\left(E_{\mathrm{F}}\right) \Delta E_{\mathrm{F}}$. and, using this in Eq. (17)

$$
\Delta E_{\mathrm{F}} \approx \frac{1}{3}\left(\Delta W-\Delta V_{\mathrm{c}}\right) .
$$

For the collapsed nanotube with band gap, we may use Eq. (11) to have

$$
\Delta E_{\mathrm{F}}+2 \Delta E_{\mathrm{F}}\left\{1-\left[\frac{E_{\mathrm{g}} / 2}{\Delta E_{\mathrm{F}}}\right]^{2}\right\}=\Delta W-\Delta V_{\mathrm{c}} \quad\left(\left|\Delta E_{\mathrm{F}}\right|>E_{\mathrm{g}} / 2\right) .
$$

We note that $\Delta V_{\mathrm{tr}}$ in the present model is given by [see Eq. (A7) in Appendix]

$$
\Delta V_{\mathrm{tr}}=2 \frac{N_{\mathrm{tr}}\left(\Delta E_{\mathrm{F}}\right)}{\rho_{\mathrm{NT}}\left(E_{\mathrm{F}}\right)}
$$

with $\alpha=2 / \rho_{\mathrm{NT}}\left(E_{\mathrm{F}}\right)$, and is twice as large as the Fermi-level shift itself for a nanotube with flat DOS in the neighborhood of the Fermi point.

The large Fermi-level shifts predicted by the DFT (LDA) calculations for the systems of NT/Au(100) can be explained in terms of Eq. (17). The nanotube DOSs obtained by such calculations (Figs. 5 and 6) virtually vanish in the neighborhood of the Fermi point, $\varepsilon_{0}$, of the nanotube and are unrealistic. Equation (17) implies that for a system with such a DOS the doping of any type is achieved only when the appreciable DOS due to the subbands are involved, inevitably leading to a large $\Delta E_{\mathrm{F}}$ if any. Figure 6 actually illustrates such a situation for the circular-NT/Au(100), yielding a rather large value of $\Delta E_{\mathrm{F}}=0.75 \mathrm{eV}$ with $N_{\mathrm{tr}}\left(\Delta E_{\mathrm{F}}\right)=0.247$ and $\rho_{\mathrm{NT}}\left(E_{\mathrm{F}}\right)=4.33($ states $/ \mathrm{eV}) /$ supercell. In this way, the approximate DFT (LDA) calculations yield unrealistic DOS and transfer doping but we may expect that a realistic estimate of $\Delta V_{\mathrm{c}}$ is inherent in such calculations since $\Delta V_{\mathrm{c}}$ is determined by the overlap of the wave functions of the nanotube and substrate and may be insensitive to the nature of approximate calculations. With this expectation we can estimate $\Delta V_{\mathrm{c}}$, which is unknown in the present model. Using the above values for $\Delta E_{\mathrm{F}}$, $N_{\text {tr }}\left(\Delta E_{\mathrm{F}}\right)$, and $\rho_{\mathrm{NT}}\left(E_{\mathrm{F}}\right)$ together with $\Delta W=0.99 \mathrm{eV}$ in Eq. (17), we find $\Delta V_{\mathrm{c}}=0.13 \mathrm{eV}$ for circular-NT/Au(100). Similarly, we have $\Delta V_{\mathrm{c}}=0.15 \mathrm{eV}$ for the collapsed-NT/Au(100) by using $\Delta E_{\mathrm{F}}=0.64 \mathrm{eV}, \quad N_{\mathrm{tr}}\left(\Delta E_{\mathrm{F}}\right)=0.202, \quad \rho_{\mathrm{NT}}\left(E_{\mathrm{F}}\right)$ $=3.05($ states $/ \mathrm{eV}) /$ supercell, and $\Delta W=0.92 \mathrm{eV}$. Assuming that the value of $\Delta V_{\mathrm{c}}$ determined in this way is realistic, we used the result, $\Delta V_{\mathrm{c}}=0.13 \mathrm{eV}$, together with the corresponding $\Delta W(=0.99 \mathrm{eV})$ in Eq. (18) to find $\Delta E_{\mathrm{F}}=0.29 \mathrm{eV}$ and $N_{\mathrm{tr}}\left(\Delta E_{\mathrm{F}}\right)=0.84$ (states $\left./ \mathrm{eV}\right) /$ supercell for the circular-NT/Au(100) with a realistic DOS. For the collapsed-NT/Au(100), using the corresponding $\Delta V_{\mathrm{c}}$ and $\Delta W$ in Eq. (19), we had similar results, $\Delta E_{\mathrm{F}}=0.26 \mathrm{eV}$ and $N_{\text {tr }}\left(\Delta E_{\mathrm{F}}\right)=0.83($ states $/ \mathrm{eV}) /$ supercell. These results of $\Delta E_{\mathrm{F}}$ and $N_{\text {tr }}$ are summarized in Table II. The present result for the circular-NT/Au, $\Delta E_{\mathrm{F}}=0.29 \mathrm{eV}$, is well compared to the STS measurements, ${ }^{26,30} \Delta E_{\mathrm{F}}=0.2-0.3 \mathrm{eV}$ for the SWNTs with $D=10-20 \AA$, and the previous theoretical prediction, ${ }^{29}$ $\Delta E_{\mathrm{F}}=0.2 \pm 0.1 \mathrm{eV}$. A careful staring at the experimental STS spectra on the collapsed-NT/Au (Ref. 34) revealed that a dip exists in the tunneling DOS at $\sim 0.27 \mathrm{eV}$ above the Fermi level, which is probably an indication of the energy gap and shows the midgap position of the collapsed nanotube (see Fig. 6). If so, the result is in good agreement with the present prediction, $\Delta E_{\mathrm{F}}=0.26 \mathrm{eV}$. We note that the above values of $\Delta V_{\mathrm{c}}$ obtained for the nanotube adsorption on the metallic surfaces are much smaller than that for the graphene adsorption, $\Delta V_{\mathrm{c}} \sim 0.90 \mathrm{eV}$. Khomyakov et al. ${ }^{62}$ have demonstrated that $\Delta V_{\mathrm{c}}$ decays exponentially as the distance, $d$, between the atoms of the nanotube and substrate becomes large. The above difference can be explained by that the atomic interactions with large $d$, compared to the minimum value of $d \sim 3.4 \AA$, are involved in the nanotube adsorption, which substantially lowers the averaged value of $\Delta V_{\mathrm{c}}$.

For the systems of circular- and collapsed-NT/Ag(100) we find $\Delta E_{\mathrm{F}}=0.04$ and $-0.02 \mathrm{eV}$, respectively, from the DFT (LDA) calculations (Fig. 5), which also provide a model with a DOS, which does not completely vanish near the Fermi point but has finite values due to the Gaussian tails of the subbands (Fig. 6). We assumed, on the basis of this observation that Eq. (17) can be applied to the system with such a DOS. Careful analyses of such DOSs near the Fermi point yielded $N_{\mathrm{tr}}\left(\Delta E_{\mathrm{F}}\right) / \rho_{\mathrm{NT}}\left(E_{\mathrm{F}}\right)=0.036$ and $-0.026 \mathrm{eV}$ and, using these values in Eq. (17), we had $\Delta V_{\mathrm{c}}=0.14$ and $0.18 \mathrm{eV}$ for the circular-NT/Ag(100) $(\Delta W=0.22 \mathrm{eV})$ and collapsed-NT/Ag(100) $(\Delta W=0.15 \mathrm{eV})$, respectively. These results of $\Delta V_{\mathrm{c}}$ are close to those obtained for $\mathrm{NT} / \mathrm{Au}(100)$, supporting the assumption that $\Delta V_{\mathrm{c}}$ does not depend on the substrate in case of the physisorption. This assumption has actually been used in the analyses of the graphene adsorption on the metallic suefaces. ${ }^{62}$ Using these values of $\Delta V_{c}$ in Eqs. (18) and (19), in which the realistic DOSs described in the previous section are now implicit, we obtained the Fermilevel shifts, $\Delta E_{\mathrm{F}}=0.03$ and $-0.03 \mathrm{eV}$ for the circular- and collapsed-NT/Ag(100), respectively. The corresponding values of $N_{\text {tr }}$ are also very small or even negligible (see Table II). If Eq. (18) can not be applicable to the system with an almost vanishing DOS such as those for the NT/Ag(100) (Fig. 5), we can alternatively use the values of $\Delta V_{c}$ obtained for the systems of NT/Au(100) on the basis of the above assumption, and find similar results of $\Delta E_{\mathrm{F}}$. These results indicate that charge transfer virtually does not occur for the NT/Ag(100), not in agreement with the experiments with large uncertainty, ${ }^{35} \Delta E_{\mathrm{F}}=-0.5$ to $-0.1 \mathrm{eV}$. It is not clear at this stage if the disagreement comes from the present inappropriate model analyses or uncertainty of the experiments.

The number of transferred electrons, $N_{\mathrm{tr}}$, is by definition [Eq. (7)] roughly proportional to the Fermi-level shift, $\Delta E_{\mathrm{F}}$, which in turn is determined by $\Delta W-\Delta V_{\mathrm{c}}[\mathrm{Eq} .(17)]$ or is even directly proportional to $\Delta W-\Delta V_{\mathrm{c}}$ [Eq. (18)]. The situation is essentially the same in the phenomenological model of Khomyakov et al. ${ }^{62}$ On the other hand, they have argued that $N_{\text {re }}$ defined for the charge redistribution and given by Eq. (5) is roughly proportional to $\Delta V$ in the graphene adsorption $\left(\Delta V_{\mathrm{NT}}\right.$ in the present case $\left.{ }^{63}\right)$. With these points in mind, we first consider the results of $N_{\text {re }}$ obtained by the DFT cal- 
culations [Fig. 4 and Eq. (5)], which yield unrealistic DOS of the nanotube on the metallic surfaces (Figs. 5 and 6). If $\Delta E_{\mathrm{F}} \approx 0\left(\Delta V_{\mathrm{tr}} \approx 0\right)$, as actually happens to $\mathrm{NT} / \operatorname{Ag}(100)$, $\Delta V_{\mathrm{NT}} \approx \Delta W \approx 0.22 \mathrm{eV}$ from Eq. (13), whereas we find $\Delta V_{\mathrm{NT}}=\Delta W-\Delta E_{\mathrm{F}} \approx 0.24 \mathrm{eV}$ for NT/Au(100), implying similar $N_{\text {re }}$ for both systems as we actually obtained (Table II). For the nanotube with realistic DOS, which was used in the above model analyses, we have $\Delta V_{\mathrm{NT}} \sim 0.8$ and $0.0 \mathrm{eV}$ for NT/Au and NT/Ag (Table II), respectively, implying that we would have quite different charge redistributions for these systems, in contrast to the above case. Now, for each system, $N_{\text {re }}$ and $N_{\text {tr }}$ would be similar to each other and both the "charge redistribution" and "charge transfer" virtually would not occur in the case of NT/Ag. In this way, the phenomenological model can also be used for the qualitative understanding of the difference or similarity between $N_{\text {re }}$ and $N_{\text {tr }}$, depending on the situation, in the nanotube physisorption on the metallic surfaces.

\section{E. Application to the graphene adsorption}

The present phenomenological model is similar to, but somewhat different from, that used by Khomyakov et al. ${ }^{62}$ in the analyses of the graphene physisorption on the metallic surfaces. ${ }^{63}$ The potential steps, $\Delta V_{\mathrm{c}}$ and $\Delta V_{\mathrm{tr}}$, have been estimated explicitly in their model, whereas we used a variational method to determine $\Delta V_{\mathrm{tr}}$ and $\Delta E_{\mathrm{F}}$, and treated $\Delta V_{\mathrm{c}}$ as a parameter to be determined by other method. The present model was devised for the nanotube adsorption but may be better applicable to the graphene adsorption on the metallic surfaces because the potential steps are better defined for the latter. Since the graphene DOS near the conical (Dirac) point shows a linear dependence on the energy and is given by $\rho_{\mathrm{G}}(E)=D_{0}\left|E-\varepsilon_{0}\right|$, where $\varepsilon_{0}$ is now the energy of the conical point, we find $N_{\mathrm{tr}}\left(\Delta E_{\mathrm{F}}\right)=(1 / 2) \rho_{\mathrm{G}}\left(E_{\mathrm{F}}\right) \Delta E_{\mathrm{F}}$ with $\rho_{\mathrm{G}}\left(E_{\mathrm{F}}\right)=D_{0}\left|\Delta E_{\mathrm{F}}\right|$. Using these relations in Eq. (17) with $\rho_{\mathrm{NT}}$ replaced by $\rho_{\mathrm{G}}$, we have

$$
\Delta E_{\mathrm{F}}=\frac{1}{2}\left(\Delta W-\Delta V_{\mathrm{c}}\right),
$$

where the WF difference is now given by $\Delta W=W_{\mathrm{M}}-W_{\mathrm{G}}$ using the graphene WF, $W_{\mathrm{G}}$. The result in Eq. (21) can be obtained by simply replacing the factor $1 / 3$ in Eq. (18) by $1 / 2$, reflecting flat and linear behaviors of the DOSs of the nanotube and graphene. Also note that $\Delta V_{\mathrm{tr}}=\Delta E_{\mathrm{F}}$ for the graphene adsorption in our model, which is compared to the numerical results, $\Delta V_{\mathrm{tr}} \sim(2 / 3) \Delta E_{\mathrm{F}}$, in the model of Khomyakov et al. If we use $\Delta W=1.04 \mathrm{eV}$ and $\Delta V_{\mathrm{c}}=0.90 \mathrm{eV}$ in Eq. (21), the values suggested by them for the graphene/Au, we have $\Delta E_{\mathrm{F}}=0.07 \mathrm{eV}$. On the other hand, the model of Khomyakov et al. yields $\Delta E_{\mathrm{F}}=0.12 \mathrm{eV}$ using the explicitly estimated value of $\Delta V_{\mathrm{tr}}=0.08 \mathrm{eV}$. These results are somewhat smaller than $\Delta E_{\mathrm{F}}=0.19 \mathrm{eV}$ directly obtained by the $a b$ initio band-structure calculations, suggesting that an accurate estimation of $\Delta V_{\mathrm{c}}$ is crucial. If we use the $a b$ initio result, $\Delta E_{\mathrm{F}}=0.19 \mathrm{eV}$, and $\Delta W=1.04 \mathrm{eV}$ in Eq. (21), we have $\Delta V_{\mathrm{c}}=0.66 \mathrm{eV}$, somewhat smaller than the suggested value, $\Delta V_{\mathrm{c}}=0.90 \mathrm{eV}$. In the absence of accurate estimations of $\Delta V_{\mathrm{c}}$, we tested several values of $\Delta V_{\mathrm{c}}$ in both
TABLE III. Fermi-level shifts, $\Delta E_{\mathrm{F}}$, of the graphene adsorbed on the $\mathrm{Au}(111)$ and $\operatorname{Ag}(111)$ surfaces. $\Delta W=W_{\mathrm{M}}-W_{\mathrm{G}}$ and $\Delta V_{\mathrm{c}}$ are the work-function difference and the potential shift used to calculate $\Delta E_{\mathrm{F}}$ by the present model [Model I: Eq. (21)] and the model of Khomyakov et al. (Model II: Ref. 62). The results of $a b$ initio calculations for $\Delta E_{\mathrm{F}}$ (DFT: Ref. 62) are also given. We used the values of $W_{\mathrm{G}}=4.50 \mathrm{eV}, W_{\mathrm{M}}=5.54 \mathrm{eV}(\Delta W=1.04 \mathrm{eV})$ for $\mathrm{Au}$, and $W_{\mathrm{M}}=4.92(\Delta W=0.42 \mathrm{eV})$ and $4.70 \mathrm{eV}(\Delta W=0.20 \mathrm{eV})$ for $\mathrm{Ag}$. Three values of $\Delta V_{\mathrm{c}}$ are tested for each case.

\begin{tabular}{lccccc}
\hline \hline & & \multicolumn{5}{c}{$\Delta E_{\mathrm{F}}$} \\
& & & \multicolumn{3}{c}{$(\mathrm{eV})$} \\
\cline { 4 - 5 } Surface & $(\mathrm{eV})$ & $(\mathrm{eV})$ & Model I & Model II & DFT \\
\hline $\mathrm{Au}(111)$ & \multirow{2}{*}{1.04} & 0.90 & 0.07 & 0.12 & 0.19 \\
& & 0.80 & 0.12 & 0.19 & \\
& & 0.70 & 0.17 & 0.25 & \\
$\mathrm{Ag}(111)$ & 0.42 & 0.90 & -0.24 & -0.33 & -0.32 \\
& & 0.80 & -0.19 & -0.27 & \\
& & 0.70 & -0.14 & -0.21 & \\
& 0.20 & 0.90 & -0.35 & -0.43 & \\
& & 0.80 & -0.30 & -0.38 & \\
& & 0.70 & -0.25 & -0.34 & \\
\hline \hline
\end{tabular}

models and the results are summarized in Table III. These analyses suggest that the values of $\Delta V_{\mathrm{c}}=0.70-0.80 \mathrm{eV}$, somewhat smaller than $0.90 \mathrm{eV}$, are more appropriate for both phenomenological models to work well. The refinement of the models might also be necessary for a better understanding, although the models have already provided useful insights into the charge transfer of the graphitic systems adsorbed on the metallic surfaces.

\section{CONCLUSIONS}

In conclusion, we have elucidated the band-gap opening by radial deformation of armchair $(21,21)$ SWNT by assuming a realistic cross-sectional shape of the collapsed nanotube. We used a semiempirical method to predict radial deformations of large SWNTs and found that the deformation of SWNTs with $D<21 \AA$ is reversible in the whole deformation range while large deformations of SWNTs with $D>21 \AA$ can be irreversible and the collapsed nanotube is stabilized without external stress. We confirmed that the collapsed armchair $(21,21)$ nanotube is a semiconductor with small band gap of $\sim 60 \mathrm{meV}$, for which hybridization of $\pi$ states on the opposing walls can be responsible as in the case of much smaller armchair SWNTs. ${ }^{25}$ Large-scale DFT calculations have been performed to clarify the charge transfer of the $(21,21)$ SWNT, both circular and collapsed, adsorbed on the $\mathrm{Au}(100)$ and $\mathrm{Ag}(100)$ surfaces. The charge redistributions obtained for the NT/Au(100) have a good correspondence, probably by happening, with the charge transfer defined by the Fermi-level shift of the nanotube adsorbed on the substrate. On the other hand, no such correspondence exists between the charge redistribution and charge transfer for the NT/Ag(100), indicating that both are different and must be 
distinguished. A phenomenological model was proposed and successfully applied to the analyses of the $a b$ initio DFT calculations for the nanotube adsorption on the metallic surfaces and to clarify the transfer doping due to such adsorptions. The Fermi-level shifts estimated on the basis of this model for the NT/Au(100) are in good agreement with the experimental observations. We found that the charge transfer virtually does not occur for the NT/Ag(100), not in agreement with the experiments with large uncertainty. We have also demonstrated that the phenomenological model can straightforwardly be applicable to the graphene adsorption on the metallic surfaces and used to make critical analyses of the previous work.

\section{ACKNOWLEDGMENTS}

This work was support by the Iketani Foundation (Grant No. 0201121-A) and a Grant-in-Aid for Scientific Research from the JSPS (Grant No. 20540394). The computations in this work were performed using the facilities of the Information Processing Center, Iwate University, and the Supercomputer Center at the ISSP, University of Tokyo. The authors acknowledge the permission to use the graphic software, VESTA.

\section{APPENDIX: OPTIMIZATION OF THE TRANSFER DOPING}

Equation (16) is conveniently rewritten as

$$
U=\int_{\varepsilon_{0}}^{E_{\mathrm{F}}}\left(E-\varepsilon_{0}\right) \rho_{\mathrm{NT}}(E) d E-N_{\mathrm{tr}}\left(\Delta E_{\mathrm{F}}\right) \Delta E_{\mathrm{F}}+N_{\mathrm{tr}}\left(\Delta E_{\mathrm{F}}\right) \Delta V_{\mathrm{tr}} .
$$

We now consider the optimization of $U$ with respect to $\Delta E_{\mathrm{F}}$ under the constraint of the Fermi-energy alignment given by Eq. (13)

$$
Y \equiv \Delta E_{\mathrm{F}}+\Delta V_{\mathrm{c}}+\Delta V_{\mathrm{tr}}-\Delta W=0 .
$$

This variational problem is solved using the Lagrange multiplier method, in which we treat $\Delta E_{\mathrm{F}}$ and $\alpha$ (in $\Delta V_{\mathrm{tr}}=\alpha N_{\mathrm{tr}}$ ) as independent variables and define a function

$$
L=U-\lambda Y,
$$

where $\lambda$ is the Lagrange multiplier. The problem now reduces to solving the equation, $\delta L=0$, under the condition $\partial L / \partial \lambda=0$. Since the derivatives, $\delta\left(\Delta E_{\mathrm{F}}\right)$ and $\delta \alpha$, are linearly independent by assumption, the vanishing of the total derivative $\delta L$ is equivalent to

$$
\frac{\partial L}{\partial \Delta E_{\mathrm{F}}}=0 \text { and } \frac{\partial L}{\partial \alpha} .
$$

Using Eqs. (A1) and (A2), and noting that $\partial N_{\mathrm{tr}} / \partial \Delta E_{\mathrm{F}}$ $=\rho_{\mathrm{NT}}\left(E_{\mathrm{F}}\right)$, we find

$$
\begin{gathered}
\frac{\partial U}{\partial \Delta E_{\mathrm{F}}}=-N_{\mathrm{tr}}\left(\Delta E_{\mathrm{F}}\right)+2 \rho_{\mathrm{NT}}\left(E_{\mathrm{F}}\right) \Delta V_{\mathrm{tr}}, \\
\frac{\partial Y}{\partial \Delta E_{\mathrm{F}}}=1+\alpha \rho_{\mathrm{NT}}\left(E_{\mathrm{F}}\right), \\
\frac{\partial U}{\partial \alpha}=N_{\mathrm{tr}}^{2}, \quad \frac{\partial Y}{\partial \alpha}=N_{\mathrm{tr}} .
\end{gathered}
$$

We use these results in Eq. (A4) to have

$$
\begin{gathered}
-N_{\mathrm{tr}}\left(\Delta E_{\mathrm{F}}\right)+2 \rho_{\mathrm{NT}}\left(E_{\mathrm{F}}\right) \Delta V_{\mathrm{tr}}-\lambda\left[1+\alpha \rho_{\mathrm{NT}}\left(E_{\mathrm{F}}\right)\right]=0, \\
N_{\mathrm{tr}}^{2}-\lambda N_{\mathrm{tr}}=0 .
\end{gathered}
$$

By eliminating $\lambda$ from these equations, we have

$$
-2 N_{\mathrm{tr}}+\rho_{\mathrm{NT}}\left(E_{\mathrm{F}}\right) \Delta V_{\mathrm{tr}}=0 .
$$

We now use the constraint of the Fermi-energy alignment, $\partial L / \partial \lambda=0$, or Eq. (A2), to eliminate $\Delta V_{\text {tr }}$ in Eq. (A7) and finally obtain

$$
\rho_{\mathrm{NT}}\left(E_{\mathrm{F}}\right)\left\{\Delta E_{\mathrm{F}}+2 \frac{N_{\mathrm{tr}}\left(\Delta E_{\mathrm{F}}\right)}{\rho_{\mathrm{NT}}\left(E_{\mathrm{F}}\right)}-\left(\Delta W-\Delta V_{\mathrm{c}}\right)\right\}=0 .
$$

This equation reduces to Eq. (17) if $\rho_{\mathrm{NT}}\left(E_{\mathrm{F}}\right)>0$. If $\rho_{\mathrm{NT}}\left(E_{\mathrm{F}}\right)=0$, which simply implies that $N_{\mathrm{tr}}=0$ [Eq. (A7)] and hence $\Delta E_{\mathrm{F}}=0$.
*Corresponding author; nisidate@iwate-u.ac.jp

${ }^{1}$ S. Iijima, Nature (London) 354, 56 (1991).

${ }^{2}$ N. Hamada, S. Sawada, and A. Oshiyama, Phys. Rev. Lett. 68, 1579 (1992).

${ }^{3}$ R. Saito, M. Fujita, G. Dresselhaus, and M. S. Dresselhaus, Appl. Phys. Lett. 60, 2204 (1992).

${ }^{4}$ J. W. Mintmire, B. I. Dunlap, and C. T. White, Phys. Rev. Lett. 68, 631 (1992).

${ }^{5}$ X. Blase, L. X. Benedict, E. L. Shirley, and S. G. Louie, Phys. Rev. Lett. 72, 1878 (1994).

${ }^{6}$ N. G. Chopra, L. X. Benedict, V. H. Crespi, M. L. Cohen, S. G. Louie, and A. Zettl, Nature (London) 377, 135 (1995).

${ }^{7}$ L. X. Benedict, V. H. Crespi, N. G. Chopra, A. Zettl, M. L. Cohen, and S. G. Louie, Chem. Phys. Lett. 286, 490 (1998).
${ }^{8}$ P. Zhang and V. H. Crespi, Phys. Rev. Lett. 83, 1791 (1999).

${ }^{9}$ D. Y. Sun, D. J. Shu, M. Ji, F. Liu, M. Wang, and X. G. Gong, Phys. Rev. B 70, 165417 (2004).

${ }^{10}$ R. B. Capaz, C. D. Spataru, P. Tangney, M. L. Cohen, and S. G. Louie, Phys. Status Solidi B 241, 3352 (2004).

${ }^{11}$ J. Zang, A. Treibergs, Y. Han, and F. Liu, Phys. Rev. Lett. 92, 105501 (2004).

${ }^{12}$ M. Hasegawa and K. Nishidate, Phys. Rev. B 74, 115401 (2006).

${ }^{13}$ C. J. Park, Y. H. Kim, and K. J. Chang, Phys. Rev. B 60, 10656 (1999).

${ }^{14}$ M. S. C. Mazzoni and H. Chacham, Appl. Phys. Lett. 76, 1561 (2000).

${ }^{15}$ P. E. Lammert, P. Zhang, and V. H. Crespi, Phys. Rev. Lett. 84, 
2453 (2000).

${ }^{16}$ C. Kilic, S. Ciraci, O. Gülseren, and T. Yildirim, Phys. Rev. B 62, R16345 (2000).

${ }^{17}$ H. S. Sim, C. J. Park, and K. J. Chang, Phys. Rev. B 63, 073402 (2001).

${ }^{18}$ O. Gülseren, T. Yildirim, S. Ciraci, and C. Kilic, Phys. Rev. B 65, 155410 (2002).

${ }^{19}$ B. Shan, G. W. Lakatos, S. Peng, and K. Cho, Appl. Phys. Lett. 87, 173109 (2005).

${ }^{20}$ D. Chen, T. Sasaki, J. Tang, and L. C. Qin, Phys. Rev. B 77, 125412 (2008).

${ }^{21}$ K. Nishidate and M. Hasegawa, Phys. Rev. B 78, 195403 (2008).

${ }^{22}$ L. Vitali, M. Burghard, P. Wahl, M. A. Schneider, and K. Kern, Phys. Rev. Lett. 96, 086804 (2006).

${ }^{23}$ A. P. M. Barboza, A. P. Gomes, B. S. Archanjo, P. T. Araujo, A. Jorio, A. S. Ferlauto, M. S. C. Mazzoni, H. Chacham, and B. R. A. Neves, Phys. Rev. Lett. 100, 256804 (2008).

${ }^{24}$ J. Q. Lu, J. Wu, W. Duan, F. Liu, B. F. Zhu, and B. L. Gu, Phys. Rev. Lett. 90, 156601 (2003).

${ }^{25}$ H. Mehrez, A. Svizhenko, M. P. Anantram, M. Elstner, and T. Frauenheim, Phys. Rev. B 71, 155421 (2005).

${ }^{26}$ J. W. G. Wildöer, L. C. Venema, A. G. Rinzler, R. E. Smalley, and C. Dekker, Nature (London) 391, 59 (1998).

${ }^{27}$ T. W. Odom, J.-L. Huang, P. Kim, and C. M. Lieber, Nature (London) 391, 62 (1998).

${ }^{28}$ A. Rubio, D. Sanchez-Portal, E. Artacho, P. Ordejon, and J. M. Soler, Phys. Rev. Lett. 82, 3520 (1999).

${ }^{29}$ Y. Xue and S. Datta, Phys. Rev. Lett. 83, 4844 (1999).

${ }^{30}$ L. C. Venema, J. W. Janssen, M. R. Buitelaar, J. W. G. Wildöer, S. G. Lemay, L. P. Kouwenhoven, and C. Dekker, Phys. Rev. B 62, 5238 (2000).

${ }^{31}$ S. G. Lemay, J. W. Jassen, M. van den Hout, M. Mooij, M. J. Bronikowski, P. A. Willis, R. E. Smalley, L. P. Kouwenhoven, and C. Dekker, Nature (London) 412, 617 (2001).

${ }^{32}$ M. Ouyang, J.-L. Huang, C. L. Cheung, and C. M. Lieber, Science 292, 702 (2001).

${ }^{33}$ S. Okada and A. Oshiyama, Phys. Rev. Lett. 95, 206804 (2005).

${ }^{34}$ C. E. Giusca, Y. Tison, and S. R. P. Silva, Phys. Rev. B 76, 035429 (2007).

${ }^{35}$ H.-J. Shin, S. Clair, Y. Kim, and M. Kawai, Appl. Phys. Lett. 93, 233104 (2008).

${ }^{36}$ W. Orellana, R. H. Miwa, and A. Fazzio, Phys. Rev. Lett. 91, 166802 (2003).

${ }^{37}$ P. M. Albrecht and J. W. Lyding, Appl. Phys. Lett. 83, 5029 (2003).

${ }^{38}$ L. B. Ruppalt and J. W. Lyding, Nanotechnology 18, 215202 (2007).

${ }^{39}$ S. J. Sque, R. Jones, S. Öberg, and P. R. Briddon, Phys. Rev. B 75, 115328 (2007).

${ }^{40}$ M. Hasegawa and K. Nishidate, e-J. Surf. Sci. Nanotechnol. 7, 541 (2009).
${ }^{41}$ J. Kürti, V. Zolyomi, M. Kertesz, and G. Sun, New J. Phys. 5, 125 (2003).

${ }^{42}$ M. Hasegawa and K. Nishidate, Phys. Rev. B 70, 205431 (2004).

${ }^{43}$ M. Hasegawa, K. Nishidate, and H. Iyetomi, Phys. Rev. B 76, 115424 (2007).

${ }^{44}$ Originally, this critical size of SWNTs has been predicted to be $D=25 \AA$ (Ref. 12). This value is reduced to $D=21 \AA$ with the use of the atomic interactions obtained by the revised semiempirical analysis of the interlayer binding of graphite (Ref. 43).

${ }^{45}$ G. Kresse and J. Furthmüller, Phys. Rev. B 54, 11169 (1996); Comput. Mater. Sci. 6, 15 (1996).

${ }^{46}$ D. M. Ceperley and B. J. Alder, Phys. Rev. Lett. 45, 566 (1980).

${ }^{47}$ J. P. Perdew and A. Zunger, Phys. Rev. B 23, 5048 (1981).

${ }^{48}$ G. Kresse and D. Joubert, Phys. Rev. B 59, 1758 (1999).

${ }^{49}$ H. J. Monkhorst and J. D. Pack, Phys. Rev. B 13, 5188 (1976).

${ }^{50}$ A. N. Imtani and V. K. Jindal, Phys. Rev. B 76, 195447 (2007).

${ }^{51}$ N. W. Ashcroft and N. D. Mermin, Solid State Physics, 7th ed. (Wiley, New York, 1996).

${ }^{52}$ H. B. Michaelson, J. Appl. Phys. 48, 4729 (1977).

${ }^{53}$ J. Zhao, J. Han, and J. P. Lu, Phys. Rev. B 65, 193401 (2002).

${ }^{54}$ B. Shan and K. Cho, Phys. Rev. Lett. 94, 236602 (2005).

${ }^{55}$ V. Barone, J. E. Peralta, J. Uddi, and G. E. Scuseria, J. Chem. Phys. 124, 024709 (2006).

${ }^{56}$ W. S. Su, T. C. Leung, and C. T. Chan, Phys. Rev. B 76, 235413 (2007).

${ }^{57}$ M. Shiraishi and M. Ata, Carbon 39, 1913 (2001).

${ }^{58}$ K. Okazaki, Y. Nakato, and K. Murakoshi, Phys. Rev. B 68, 035434 (2003)

${ }^{59}$ S. Suzuki, Y. Watanabe, Y. Homma, S. Fukuba, S. Heun, and A. Locatelli, Appl. Phys. Lett. 85, 127 (2004).

${ }^{60}$ The use of only the $\Gamma$ point in the supercell calculations implies that five $k$ points are effectively taken for the nanotube because the supercell contains five unit cells of the nanotube. However, any of these $k$ points is away from $k_{0} \approx(1 / 3)(2 \pi / a)$, the wave number at the Fermi point (see Fig. 2), indicating that the linearband contributions to the DOS near the Fermi point are not properly taken into account. We also note that, since the nanotube DOS itself near the Fermi point is very small, its modification, if any, may be quite small and can only be detected only by very accurate calculations.

${ }^{61}$ C. T. White and J. W. Mintmire, J. Phys. Chem. B 109, 52 (2005).

${ }^{62}$ P. A. Khomyakov, G. Giovannetti, P. C. Rusu, G. Brocks, J. van den Brink, and P. J. Kelly, Phys. Rev. B 79, 195425 (2009).

${ }^{63}$ In case of the graphene adsorption, we use the second equation, $W_{\mathrm{M}}=W+\Delta V$, in Eq. (13), which is the definition of $\Delta V$ (Ref. 62 ), and $\Delta V$ is now given by $\Delta V_{\mathrm{NT}}$ and $W$ by $W_{\mathrm{G}}+\Delta E_{\mathrm{F}}$ (the Fermi-energy alignment). Hence, the present phenomenological model can straightforwardly be applicable to the graphene adsorption by simply replacing $W_{\mathrm{NT}}$ by the graphene $\mathrm{WF}, W_{\mathrm{G}}$. 